\title{
Effects of Beetroot Juice Supplementation on Cardiorespiratory Endurance in Athletes. A Systematic Review
}

\author{
Raúl Domínguez ${ }^{1}$, Eduardo Cuenca ${ }^{2}$, José Luis Maté-Muñoz ${ }^{1}$, Pablo García-Fernández ${ }^{1}$, \\ Noemí Serra-Paya ${ }^{2}$, María Carmen Lozano Estevan ${ }^{1}$, Pablo Veiga Herreros ${ }^{1}$ \\ and Manuel Vicente Garnacho-Castaño ${ }^{2, *}$ \\ 1 College of Health Sciences, University Alfonso X El Sabio University, Madrid 29651, Spain; \\ rdomiher@uax.es (R.D.); jmatmuo@uax.es (J.L.M.-M.); pablgafe@uax.es (P.G.-F.); \\ mloza@myuax.com (M.C.L.E.); pveigher@uax.es (P.V.H.) \\ 2 Tecnocampus, College of Health Sciences, University of Pompeu Fabra, Mataró-Maresme, \\ Barcelona 08302 Spain; educuen@hotmail.com (E.C.); nserra@tecnocampus.cat (N.S.-P.) \\ * Correspondence: mgarnacho@escs.tecnocampus.cat
}

Received: 21 October 2016; Accepted: 30 December 2016; Published: 6 January 2017

\begin{abstract}
Athletes use nutritional supplementation to enhance the effects of training and achieve improvements in their athletic performance. Beetroot juice increases levels of nitric oxide (NO), which serves multiple functions related to increased blood flow, gas exchange, mitochondrial biogenesis and efficiency, and strengthening of muscle contraction. These biomarker improvements indicate that supplementation with beetroot juice could have ergogenic effects on cardiorespiratory endurance that would benefit athletic performance. The aim of this literature review was to determine the effects of beetroot juice supplementation and the combination of beetroot juice with other supplements on cardiorespiratory endurance in athletes. A keyword search of DialNet, MedLine, PubMed, Scopus and Web of Science databases covered publications from 2010 to 2016. After excluding reviews/meta-analyses, animal studies, inaccessible full-text, and studies that did not supplement with beetroot juice and adequately assess cardiorespiratory endurance, 23 articles were selected for analysis. The available results suggest that supplementation with beetroot juice can improve cardiorespiratory endurance in athletes by increasing efficiency, which improves performance at various distances, increases time to exhaustion at submaximal intensities, and may improve the cardiorespiratory performance at anaerobic threshold intensities and maximum oxygen uptake $\left(\mathrm{VO}_{2 \max }\right)$. Although the literature shows contradictory data, the findings of other studies lead us to hypothesize that supplementing with beetroot juice could mitigate the ergolytic effects of hypoxia on cardiorespiratory endurance in athletes. It cannot be stated that the combination of beetroot juice with other supplements has a positive or negative effect on cardiorespiratory endurance, but it is possible that the effects of supplementation with beetroot juice can be undermined by interaction with other supplements such as caffeine.
\end{abstract}

Keywords: nutrition; sport; exercise; nitric oxide; physical activity

\section{Introduction}

Cardiorespiratory endurance is defined as a health-related component of physical fitness that relates to the ability of the circulatory and respiratory systems to supply fuel during sustained physical activity and to eliminate fatigue products after supplying fuel [1]. Cardiorespiratory endurance is a performance factor in all sports in which adenosine triphosphate (ATP) is resynthesized, mainly by aerobic metabolism or oxidative processes that produce energy. In these sports, the expended effort typically lasts longer than five minutes, primarily depending on the metabolic level of the oxidative 
processes involved [2]. Factors that limit performance in this type of endurance patterns include maximum oxygen uptake $\left(\mathrm{VO}_{2 \max }\right)$, ventilatory thresholds (first and second ventilatory threshold) and energy efficiency or economy [3-5].

In competitive sports, $0.5 \%-1.5 \%$ improvements in performance are considered a critical difference [6]. In order to enhance the effects of training and improve performance, athletes often turn to nutritional supplements [7]. According to the American College of Sports Medicine (ACSM), adequate selection of nutrients and supplements, adjusting intake according to the exercise performed, is necessary for optimal performance in athletes [8]. However, not all supplements have been shown to produce a positive effect on performance. The Australian Institute of Sport [9], classified supplements to which athletes have access, with the goal of categorizing nutritional supplements based on the level of evidence for impact on an athlete's performance (Table 1). However, the effectiveness of supplements also depends on dosage and type of effort, because the potential ergogenic effect may differ by the specific type of sport [10].

Table 1. Classification of nutritional supplements, based on performance effect. Adapted from Australian Institute of Sport [9] and Burke [11].

\begin{tabular}{|c|c|c|}
\hline Category & Sub-Categories & Supplements \\
\hline High level of evidence & $\begin{array}{c}\text { Will improve athletic performance } \\
\text { with adequate dosing and specific } \\
\text { types of effort }\end{array}$ & $\begin{array}{c}\beta \text {-alanine } \\
\text { Sodium bicarbonate } \\
\text { Caffeine } \\
\text { Creatinine } \\
\text { Beetroot juice }\end{array}$ \\
\hline $\begin{array}{l}\text { Moderate level } \\
\text { of evidence }\end{array}$ & $\begin{array}{l}\text { May improve performance, under } \\
\text { specific dosing and effort } \\
\text { conditions, although additional } \\
\text { research is needed }\end{array}$ & $\begin{array}{c}\text { Fish oils } \\
\text { Carnitine } \\
\text { Curcumin } \\
\text { Glucosamine } \\
\text { Glutamine } \\
\text { HMB } \\
\text { Quercetin } \\
\text { Vitamins } C \text { and E } \\
\text { Tart cherry juice }\end{array}$ \\
\hline Low level of evidence & No demonstrated beneficial effects & Supplements not found in other categories \\
\hline Prohibited supplements & $\begin{array}{l}\text { May result in positive doping tests } \\
\text { and therefore are prohibited }\end{array}$ & $\begin{array}{l}\text { Substances on the list published annually } \\
\text { by the World Anti-Doping Agency (WADA) }\end{array}$ \\
\hline
\end{tabular}

Beetroot juice is used as a supplement because of its high inorganic nitrate $\left(\mathrm{NO}_{3}{ }^{-}\right)$content, a compound found naturally in vegetables and in processed meats, where it is used as a preservative [12].

Once ingested, the $\mathrm{NO}_{3}{ }^{-}$is reduced to nitrite $\left(\mathrm{NO}_{2}{ }^{-}\right)$, by anaerobic bacteria in the oral cavity by the action of nitrate reductase enzymes [13] and then to nitric oxide (NO) in the stomach [14]. This physiological mechanism depends on the entero-salivary circulation of inorganic nitrate without involving NOS activity. Once in the acidic stomach, nitrite is instantly decomposed to convert to NO and other nitrogen oxides performing determinant physiological functions (Figure 1). Nitrate and remaining nitrite is absorbed from the intestine into the circulation, which can become bioactive NO in tissues and blood [14] under physiological hypoxia.

$\mathrm{NO}$ induces several physiological mechanisms that influences $\mathrm{O}_{2}$ utilization during contraction skeletal muscle. Physiological mechanisms for $\mathrm{NO}_{2}{ }^{-}$reduction are facilitated by hypoxic conditions, therefore, $\mathrm{NO}$ (vasodilator) is produced in those parts of muscle that are consuming or in need of more $\mathrm{O}_{2}$. This mechanism would allow local blood flow to adapt to $\mathrm{O}_{2}$ requirement providing within skeletal muscle an adequate homogeneous distribution. This physiological response could be positive in terms of muscle function, although it would not explain a reduced $\mathrm{O}_{2}$ cost during exercise [15]. Another probable mechanism is related to $\mathrm{NO}_{2}{ }^{-}$and $\mathrm{NO}$ as regulators of cellular $\mathrm{O}_{2}$ utilization [15]. 


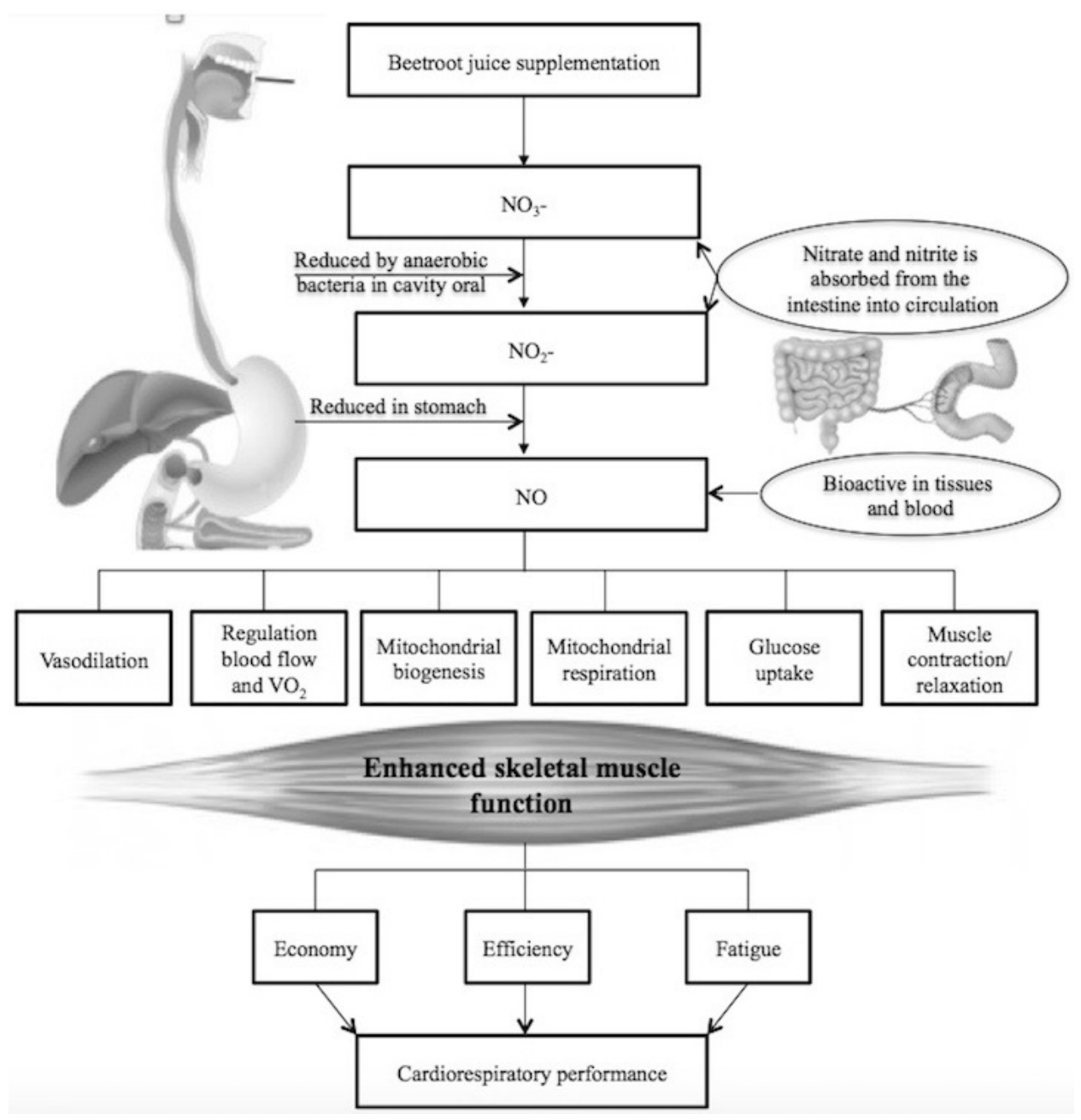

Figure 1. Pathway of nitric oxide (NO) production from beetroot juice supplementation. Nitrate $\left(\mathrm{NO}_{3}{ }^{-}\right)$is reduced to nitrite $\left(\mathrm{NO}_{2}{ }^{-}\right)$by anaerobic bacteria in the oral cavity and then to $\mathrm{NO}$ in the stomach. $\mathrm{NO}_{3}{ }^{-}$and remaining $\mathrm{NO}_{2}{ }^{-}$are absorbed from the intestine into the circulation, which can become bioactive NO in tissues and blood. NO induces several physiological functions improving skeletal muscle function and, consequently, increasing cardiorespiratory performance.

In addition, a potent signaling molecule that affects cell function in many body tissues, NO is endogenously produced by synthesizing nitric oxide from L-arginine oxidation. The molecule has important hemodynamic and metabolic functions [16,17], being a major vasodilator that can increase blood flow to muscles [18] and promote oxygen transfer in the muscle. Additional physiological benefits of NO include improved mitochondrial efficiency and glucose uptake in muscle [19] and enhanced muscle contraction and relaxation processes [20]. Other researchers have reported that NO can act as an immunomodulator [21] and stimulates gene expression and mitochondrial biogenesis [22]. Given the positive effects of beetroot juice, which are induced by means of NO, this supplement has been proposed as part of the therapeutic approach in people with chronic obstructive pulmonary disease [23], hypertension [24], heart failure [25] and insulin resistance [26].

These findings reflect the importance of supplementation with $\mathrm{NO}_{3}{ }^{-}$or nitrate salts to increase the bioavailability of $\mathrm{NO}$ in order to influence muscle function improving exercise performance, mainly in aerobic metabolism [27]. Therefore, supplementation with beetroot juice may have an ergogenic effect in athletes [9], especially with respect to cardiorespiratory endurance. However, the assumption that the beetroot juice supplementation improves performance in cardiorespiratory endurance under 
hypoxic conditions, and the combination of beetroot juice supplementation with other supplements, as caffeine, has a positive effect on cardiorespiratory endurance is controversial.

The objective of the present literature review was to analyze the effects of beetroot juice supplementation on cardiorespiratory endurance in several conditions (normoxia, hypoxia and beetroot juice with other supplements) and determine the appropriate dosage to enhance the potential ergogenic effects on performance. The focus of the article is mainly on the influence of beetroot juice of the acute and chronic responses on trained endurance athletes.

\section{Methodology}

A keyword search for articles published in English or Spanish since 2010 was carried out in the DialNet, MedLine, PubMed, Scopus and Web of Science databases on 8 June 2016. The search terms included beet, beetroot, nitrate, nitrite, supplement, supplementation, nutrition, "sport nutrition" and "ergogenic aids". The 210 selected articles included at least one of those search terms, in combination with endurance, exercise, sport or athlete.

Exclusion criteria were the following: literature reviews and meta-analyses, animal studies, population other than endurance athletes, and inadequate assessment of cardiorespiratory endurance, specifically defined as $\leq \mathrm{VO}_{2 \max }$ testing or no test lasting more than 5 min to determine how long the subject can maintain the lowest intensity at which $\mathrm{VO}_{2 \max }$ was achieved [28]. Therefore, 23 articles were selected for the present review (Figure 2).

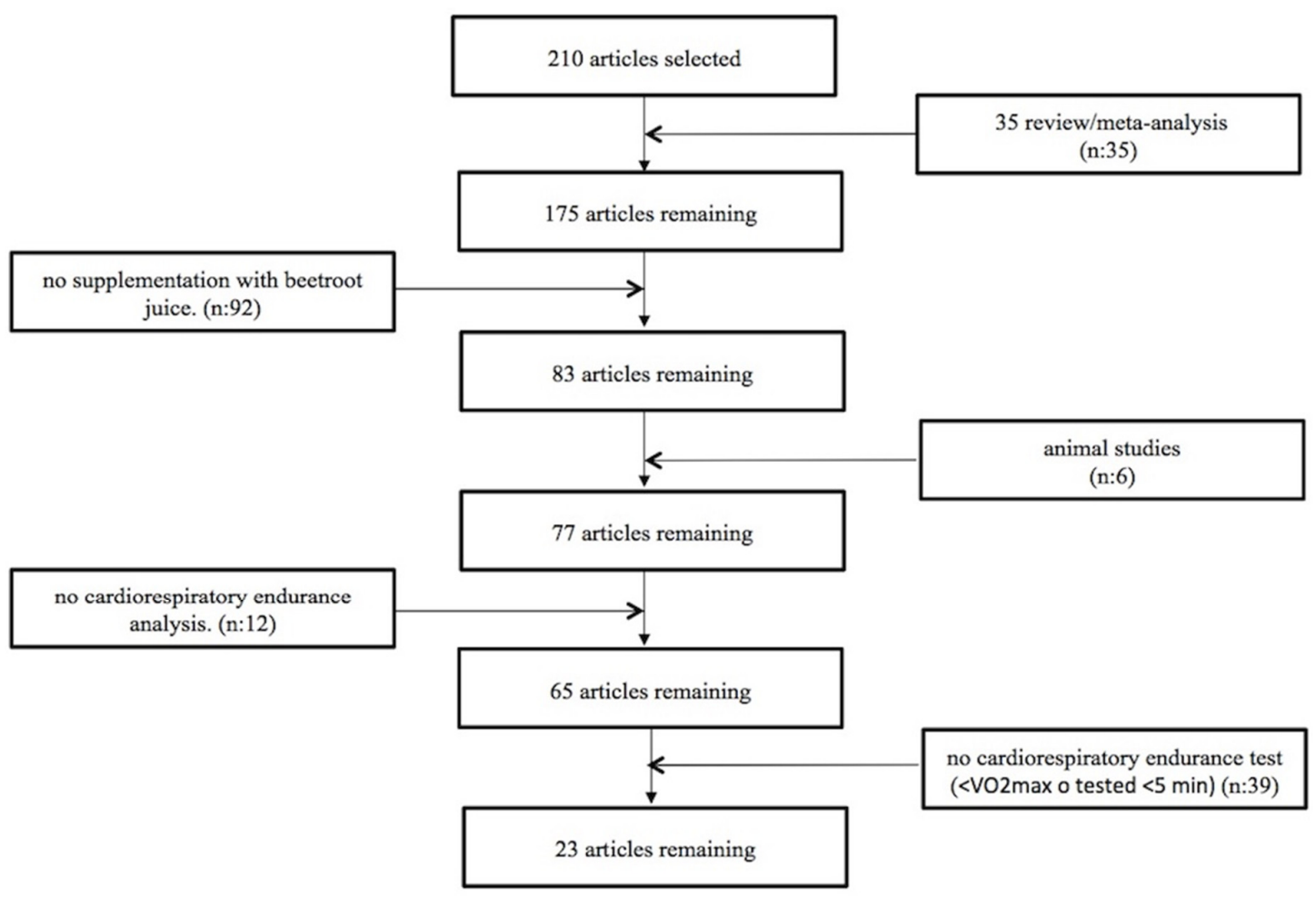

Figure 2. Flowchart of article selection.

\section{Results and Discussion}

The selected studies on the effects of beetroot juice supplementation on cardiorespiratory endurance are summarized in Table 2. 
Table 2. Summary of studies that have evaluated the performance or metabolic responses after supplementation protocol with beet juice.

\begin{tabular}{|c|c|c|c|c|c|}
\hline Reference & Participants & Experimental Conditions & $\begin{array}{l}\text { Supplementation } \\
\text { Protocol }\end{array}$ & Variables & Results \\
\hline [12] & $\begin{array}{l}\mathrm{M}(n: 5) \text { and } \mathrm{W} \\
(n: 6) \text {, trained } \\
\text { athletes }\end{array}$ & $\begin{array}{l}\text { EC1: beet juice, } \\
\text { EC2: placebo }\end{array}$ & $\begin{array}{l}\text { EC1: beet juice ( } 8 \mathrm{mmol} \\
\text { nitrate) }(90 \text { min before) }\end{array}$ & Test 5 km: Performance, HR, RPE & $\begin{array}{l}\text { Performance: Last mile 1.1: faster EC1 vs. EC2 (5\%), } \\
\text { RPE: } 1 \text { mile: lower in EC1 vs. EC2 }\end{array}$ \\
\hline [27] & $\begin{array}{l}\text { M, competitive } \\
\text { cyclists }(n: 9)\end{array}$ & $\begin{array}{l}\text { EC1: beet juice, } \\
\text { EC2: placebo }\end{array}$ & $\begin{array}{l}\text { EC1: } 500 \mathrm{~mL} \text { beet juice } \\
(6.2 \mathrm{mmol} \text { nitrate) } \\
\text { (120 min before) }\end{array}$ & $\begin{array}{l}\text { Tests } 4 \mathrm{~km} \text { and } 16 \mathrm{~km} \text { : respiratory } \\
\text { parameters, performance }\end{array}$ & 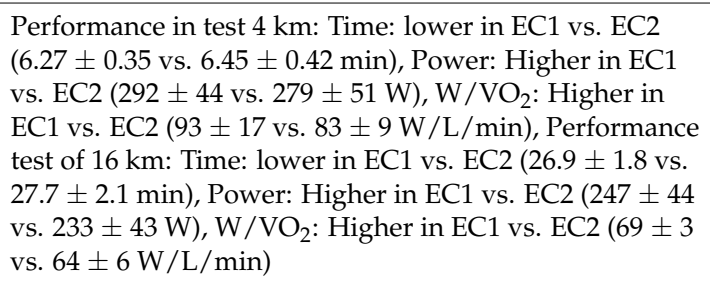 \\
\hline [29] & $\begin{array}{l}\text { M, national level } \\
\text { athletes kayak } \\
(n: 5)\end{array}$ & $\begin{array}{l}\text { EC1: beet juice, } \\
\text { EC2: placebo }\end{array}$ & $\begin{array}{l}\text { Study A: EC1: } 140 \mathrm{~mL} \\
\text { beet juice ( } 4.8 \mathrm{mmol} \\
\text { nitrate) }(150 \mathrm{~min} \text { before), } \\
\text { Study B: EC1: } 140 \mathrm{~mL} \text { beet } \\
\text { juice ( } 9.6 \mathrm{mmol} \text { nitrate) } \\
\text { (150 min before) }\end{array}$ & $\begin{array}{l}\text { Study A: kayaking incremental test: } \\
\text { Test } 10 \text { min (10 min }+5 \text { min LT1 } \\
\text { LT2) + } 4 \text { min test: respiratory } \\
\text { parameters, lactate, performance } \\
\text { (test } 4 \text { min), HR, RPE. Study B: Test } \\
500 \mathrm{~m}\end{array}$ & $\begin{array}{l}\text { Study A: } 4 \text { min test: } \mathrm{VO}_{2}: \text { decreases in EC1 vs. EC2 } \\
\text { ( } 46.87 \pm 2.56 \mathrm{vs} .47 .83 \pm 2.77 \mathrm{~mL} / \mathrm{kg} / \mathrm{min}), \text { Economy: } \\
\text { improved } \mathrm{EC} 1 \mathrm{vs} . \mathrm{EC} 2(189.67 \pm 8.17 \mathrm{vs} \text {. } \\
193.90 \pm 8.17 \mathrm{~mL} / \mathrm{kg} / \mathrm{km}) . \text { Study B: Test } 500 \mathrm{~m} \text { : Time: } \\
\text { improved } \mathrm{EC} 1 \text { vs. EC2 }(114.6+1.5 \mathrm{~s} \text { vs. } 116.7+2.2 \mathrm{~s}) \text {, } \\
\text { Rowing often partially } 100-400 \mathrm{~m} \text { : increases in EC1 vs. } \\
\text { EC2 (108 + } 2 \text { vs. } 105+2 \text { strokes), Partial speed } 100-400 \mathrm{~m}: \\
\text { increases in EC1 vs. EC2 }(4.40+0.03 \text { vs. } 4.30+0.05 \mathrm{~m} / \mathrm{s})\end{array}$ \\
\hline$[30]$ & $\begin{array}{l}\text { M, trained } \\
\text { cyclists-triathletes } \\
(n: 13)\end{array}$ & $\begin{array}{l}\text { EC1: beet juice, } \\
\text { EC2: placebo }\end{array}$ & $\begin{array}{l}\text { EC1: beet juice } 140 \mathrm{~mL} \\
\text { ( } 8 \mathrm{mmol} \text { nitrate) }(6 \text { days })\end{array}$ & $\begin{array}{l}\text { Test } 30 \mathrm{~min} \text { at } 45 \% \mathrm{MAP}+30 \text { min at } \\
65 \% \mathrm{MAP}+\text { test } 10 \mathrm{~km} \text { : respiratory } \\
\text { parameters, lactate, glucose, } \\
\text { performance (test to exhaustion at } \\
\left.80 \% \mathrm{VO}_{2 \max }\right), \mathrm{HR}, \mathrm{RPE}\end{array}$ & $\begin{array}{l}\text { Respiratory parameters } \mathrm{VO}_{2} \text { at } 45 \% \mathrm{MAP} \text { : lower in } \mathrm{EC} 1 \\
\text { vs. EC2 }(1.93 \pm 0.05 \text { vs. } 2.0 \pm 0.07 \mathrm{~L} / \mathrm{min}), \mathrm{VO}_{2} \text { at } 65 \% \\
\mathrm{MAP}: \text { lower in } \mathrm{EC} 1 \text { vs. } \mathrm{EC} 2(2.94 \pm 0.10 \mathrm{vs} \text {. } \\
3.1 \pm 0.09 \mathrm{~L} / \mathrm{min}), \text { Performance (test } 10 \mathrm{~km}) \text { : } \\
\text { improvement in EC1 vs. } \mathrm{EC} 2(953 \pm 21 \mathrm{vs.} 965 \pm 21 \mathrm{~s})\end{array}$ \\
\hline [31] & $\begin{array}{l}\text { M, trained } \\
\text { athletes }(n: 13)\end{array}$ & $\begin{array}{l}\text { EC1: beet juice, } \\
\text { EC2: placebo }\end{array}$ & $\begin{array}{l}\text { EC1: } 280 \mathrm{~mL} \text { of beet juice } \\
\text { (6.5 mmol nitrate) for } \\
7 \text { days. EC2: Control }\end{array}$ & $\begin{array}{l}\text { Test } 20 \min (10 \min \text { to } 10 \mathrm{~min} \\
\left.50 \%+70 \% \mathrm{VO}_{2 \max }\right): \\
\text { respiratory parameters }\end{array}$ & $\begin{array}{l}\text { Respiratory parameters: } 70 \% \mathrm{VO}_{2 \max } \text { : oxygen } \\
\text { consumption decrease in } \mathrm{EC} 1(3 \%)\end{array}$ \\
\hline [32] & $\begin{array}{l}\text { M, trained } \\
\text { cyclists }(n: 8)\end{array}$ & $\begin{array}{l}\text { EC1: beet juice, } \\
\text { EC2: placebo }\end{array}$ & $\begin{array}{l}\text { EC1: } 500 \mathrm{~mL} \text { beet juice } \\
(6.2 \mathrm{mmol} \text { nitrate) } \\
\text { (150 min before) }\end{array}$ & $\begin{array}{l}\text { Test } 50 \text { miles: respiratory } \\
\text { parameters, lactate, performance }\end{array}$ & $\begin{array}{l}\text { Performance: last } 10 \text { miles: lower time in EC1 vs. EC2, } \\
\mathrm{W} / \mathrm{VO}_{2}: \text { higher in EC1 vs. EC2 }(67.4 \pm 5.5 \mathrm{vs.} \\
65.3 \pm 4.8 \mathrm{~W} / \mathrm{L} / \mathrm{min})\end{array}$ \\
\hline [33] & $\begin{array}{l}\text { M, trained } \\
\text { athletes }(n: 16)\end{array}$ & $\begin{array}{l}\text { EC1: beet juice, } \\
\text { EC2: placebo }\end{array}$ & $\begin{array}{l}\text { EC1: } 450 \mathrm{~mL} \text { beet juice } \\
\text { ( } 5 \mathrm{mmol} \text { nitrate) (115 min } \\
\text { before) }\end{array}$ & $\begin{array}{l}\text { Test } 40 \min \left[20 \min \text { at } 50 \% \mathrm{VO}_{2 \max }\right. \\
\left.+20 \text { min at } 70 \% \mathrm{VO}_{2 \max }\right]+ \text { time to } \\
\text { exhaustion at } 90 \% \mathrm{VO}_{2 \max } \text { : } \\
\text { respiratory parameters, } \\
\text { performance (test to exhaustion at } \\
90 \% \mathrm{VO}_{2 \max } \text { ), lactate, } \mathrm{HR}, \mathrm{RPE}\end{array}$ & $\begin{array}{l}\text { Respiratory parameters: RER: greater in EC1 vs. EC2 at } \\
50 \% \mathrm{VO}_{2 \max }(0.89 \pm 0.03 \text { vs. } 0.86 \pm 0.06) \text { and test to } \\
\text { exhaustion }(1.04 \pm 0.06 \text { vs. } 1.01 \pm 0.06) \text {, Performance (test } \\
\left.\text { to exhaustion at } 90 \% \mathrm{VO}_{2 \max }\right) \text { : time increases in EC1 vs. } \\
\mathrm{EC} 2(185 \pm 122 \mathrm{~s} \text { vs. } 160 \pm 109 \mathrm{~s}), \text { Max lactate: Higher in } \\
\mathrm{EC} 1 \text { vs. } \mathrm{EC} 2(8.80 \pm 2.10 \mathrm{vs} .7 .90 \pm 2.30 \mathrm{mmol} / \mathrm{L})\end{array}$ \\
\hline
\end{tabular}


Table 2. Cont.

\begin{tabular}{|c|c|c|c|c|c|}
\hline Reference & Participants & Experimental Conditions & $\begin{array}{l}\text { Supplementation } \\
\text { Protocol }\end{array}$ & Variables & Results \\
\hline [34] & $\begin{array}{l}\text { M, kayakers } \\
(n: 8)\end{array}$ & $\begin{array}{l}\text { EC1: beet juice, } \\
\text { EC2: placebo }\end{array}$ & $\begin{array}{l}\text { CE1: beet juice } 70 \mathrm{~mL} \\
(5 \mathrm{mmol} \text { nitrate }) \\
(180 \mathrm{~min} \text { before })\end{array}$ & $\begin{array}{l}\text { Test } 15 \text { min at } 60 \% \text { MAP }+5 \times 10 \mathrm{~s} \text {. } \\
\text { R: } 50 \mathrm{~s}+5 \text { min recovery }+ \text { Test } 1 \mathrm{~km} \\
\text { kayak: respiratory parameters, } \\
\text { performance }(5 \times 10 \mathrm{~s}) \\
\text { performance }(1 \mathrm{~km} \text { time trial), HR }\end{array}$ & $\begin{array}{l}\text { Test } 15 \text { min at } 60 \% \text { MAP: respiratory parameters: } \mathrm{VO}_{2} \\
\text { lower in } \mathrm{EC} 1 \mathrm{vs} \text {. EC2 }(35.6 \pm 2.5 \mathrm{vs} \text {. } \\
36.8 \pm 2.4 \mathrm{~mL} / \mathrm{kg} / \mathrm{min}), \text { Test } 1 \mathrm{~km} \text { : respiratory } \\
\text { parameters: } \mathrm{VO}_{2} \text { lower in } \mathrm{EC} 1 \mathrm{vs} \text {. EC2 (results } \\
\text { not specified) }\end{array}$ \\
\hline [35] & $\begin{array}{l}\text { M, trained } \\
\text { athletes }(n: 9)\end{array}$ & $\begin{array}{l}\text { EC1: beet juice, } \\
\text { EC2: placebo }\end{array}$ & $\begin{array}{l}\text { EC1: } 500 \mathrm{~mL} \text { beet juice } \\
(8.2 \mathrm{mmol} \text { nitrate })\end{array}$ & $\begin{array}{l}\text { Tests to exhaustion at } 60 \%, 70 \% \text {, } \\
80 \% \text { and } 100 \% \mathrm{VO}_{2 \max }: \text { respiratory } \\
\text { parameters, lactate, } \\
\text { performance, } \mathrm{HR}\end{array}$ & $\begin{array}{l}\text { Performance: } 60 \% \mathrm{VO}_{2 \max }: \mathrm{EC} 1 \text { more time to exhaustion } \\
\text { vs. EC2 ( } 696 \pm 120 \text { vs. } 593 \pm 68 \mathrm{~s}), 70 \% \mathrm{VO}_{2 \max } \text { : EC1 } \\
\text { more time to exhaustion vs. EC2 }(452 \pm 106 \text { vs. } \\
390 \pm 86 \mathrm{~s}), 80 \% \mathrm{VO}_{2 \max }: \mathrm{EC} 1 \text { more time to exhaustion vs. } \\
\mathrm{EC} 2(294 \pm 50 \text { vs. } 263 \pm 50 \mathrm{~s})\end{array}$ \\
\hline [36] & $\begin{array}{l}\mathrm{M}(n: 5) \text { and } \mathrm{W} \\
(n: 3) \text {, trained } \\
\text { athletes }(n: 8)\end{array}$ & $\begin{array}{l}\text { EC1: beet juice, } \\
\text { EC2: placebo }\end{array}$ & $\begin{array}{l}\text { EC1: beet juice } 500 \mathrm{~mL} \\
(5.2 \text { mmol nitrate) } \\
\text { (15 days) }\end{array}$ & $\begin{array}{l}\text { Test } 5 \text { min at } 90 \% \text { VT } 1+ \\
\text { incremental test: respiratory } \\
\text { parameters, lactate, performance, } \\
\text { HR, glucose }\end{array}$ & $\begin{array}{l}\text { Respiratory parameters: Test } 5 \mathrm{~min} \text { at } 90 \% \text { VT1 }(\text { day } 15) \text { : } \\
\mathrm{VO}_{2} \text { lower in } \mathrm{EC} 1 \text { vs. EC2 ( } 1.37 \pm 0.23 \text { vs. } 1.43 \pm 0.23 \\
\mathrm{~L} / \mathrm{min}) \text {, Incremental test: Wpeak: Higher EC } 1 \text { vs. EC2 } \\
(331 \pm 68 \text { vs. } 323 \pm 68 \mathrm{~W}) \text {, WVT1: Higher EC1 vs. EC2 } \\
(105 \pm 28 \text { vs. } 84 \pm 18 \mathrm{~W})\end{array}$ \\
\hline [37] & $\begin{array}{l}\mathrm{M}(n: 4) \text { and } \mathrm{W} \\
(n: 5), \text { Healthy, } \\
\text { physically active } \\
\text { participants }\end{array}$ & $\begin{array}{l}\text { EC1: beet juice, } \\
\text { EC2: placebo }\end{array}$ & $\begin{array}{l}\text { EC1: beet juice } 140 \mathrm{~mL} \\
\text { ( } 8 \mathrm{mmol} \text { nitrate) (6 days) }\end{array}$ & $\begin{array}{l}\text { Test } 4 \text { min at } 90 \% \text { VT1 + test to } \\
\text { exhaustion at } 70 \% \text { between VT1 } \\
\text { and } \mathrm{VO}_{2 \max } \text { : respiratory } \\
\text { parameters, lactate, performance } \\
\text { (test to exhaustion at } 70 \% \text { between } \\
\left.\text { VT1 and } \mathrm{VO}_{2 \max }\right), \mathrm{HR}\end{array}$ & $\begin{array}{l}\text { Performance (test to exhaustion at } 70 \% \text { between VT1 and } \\
\left.\mathrm{VO}_{2 \text { max }}\right) \text { higher EC } 1 \text { vs. EC2 }(635 \pm 258 \text { vs. } 521 \pm 158 \text { s) }\end{array}$ \\
\hline [38] & $\begin{array}{l}\text { M, trained } \\
\text { swimmers (n: 14) }\end{array}$ & $\begin{array}{l}\text { EC1: beet juice, } \\
\text { EC2: placebo }\end{array}$ & $\begin{array}{l}\text { EC1: beet juice } 500 \mathrm{~mL} \\
\text { ( } 5.5 \text { mmol nitrate) ( } 6 \text { days), } \\
\text { EC2: placebo ( } 6 \text { days) }\end{array}$ & Incremental test in swimming & $\begin{array}{l}\text { VT1: improvement in EC1 vs. EC2 }(6.7 \pm 1.2 \text { vs. } \\
6.3 \pm 1.0 \mathrm{~kg}) \text {, energy expenditure: decreases in } \mathrm{EC} 1 \mathrm{vs.} \\
\mathrm{EC} 2(1.7 \pm 0.3 \text { vs. } 1.9 \pm 0.5 \mathrm{kcal} / \mathrm{kg} / \mathrm{h})\end{array}$ \\
\hline [39] & $\begin{array}{l}\text { M, trained } \\
\text { cyclists-triathletes } \\
(n: 9)\end{array}$ & $\begin{array}{l}\text { EC1: hypoxia }(2500 \mathrm{~m})+ \\
\text { beet juice, EC2: hypoxia } \\
(2500 \mathrm{~m})+\text { placebo }\end{array}$ & $\begin{array}{l}\text { EC1: beet juice } 70 \mathrm{~mL} \\
\text { ( } 5 \text { mmol nitrate) }(150-180 \\
\text { min before) }\end{array}$ & $\begin{array}{l}\text { Test } 15 \text { min at } 60 \% \mathrm{VO}_{2 \max }+\text { test of } \\
16.1 \mathrm{~km} \text { in hypoxia }(2500 \mathrm{~m}): \\
\text { respiratory parameters, lactate, } \\
\text { performance }(16.1 \mathrm{~km} \text { time trial })\end{array}$ & $\begin{array}{l}\text { Test } 15 \text { min at } 60 \% \mathrm{VO}_{2 \max } \text { : respiratory parameters: } \mathrm{VO}_{2} \\
\text { lower in EC1 vs. EC2 (improvement unspecified), } \\
\text { performance ( } 16.1 \mathrm{~km} \text { time trial): Time: improved } \mathrm{EC} 1 \text { vs. } \\
\text { EC2 }(1664 \pm 14 \mathrm{vs} .1716 \pm 17 \mathrm{~s}) \text {, Power: improved EC1 vs. } \\
\text { EC2 }(224 \pm 6 \text { vs. } 216 \pm 6 \mathrm{~W})\end{array}$ \\
\hline$[40]^{*}$ & $\begin{array}{l}\text { M, trained } \\
\text { cyclists }(n: 11)\end{array}$ & $\begin{array}{l}\text { EC1: normoxia + beet } \\
\text { juice, EC2: normoxia + } \\
\text { placebo, EC3: hypoxia } \\
(2500 \mathrm{~m})+\text { beet juice, EC4: } \\
\text { hypoxia }(2500 \mathrm{~m})+ \\
\text { placebo }\end{array}$ & $\begin{array}{l}\text { EC1: beet juice } 70 \mathrm{~mL} \\
(6.5 \mathrm{mmol} \text { nitrate })(120 \\
\text { min before), EC3: beet } \\
\text { juice } 70 \mathrm{~mL}(6.5 \mathrm{mmol} \\
\text { nitrate) }(120 \mathrm{~min} \text { before })\end{array}$ & $\begin{array}{l}\text { Test } 15 \text { min } 50 \%+\text { test MAP } 10 \mathrm{~km} \text { : } \\
\text { respiratory parameters, } \\
\text { performance }(10 \mathrm{~km}), \mathrm{HR}\end{array}$ & No differences in analyzed variables \\
\hline
\end{tabular}


Table 2. Cont.

\begin{tabular}{|c|c|c|c|c|c|}
\hline Reference & Participants & Experimental Conditions & $\begin{array}{l}\text { Supplementation } \\
\text { Protocol }\end{array}$ & Variables & Results \\
\hline [41] & $\begin{array}{l}\text { M, trained } \\
\text { runners }(n: 10)\end{array}$ & $\begin{array}{l}\text { EC1: beet juice (n: 5), } \\
\text { EC2: placebo (n: 5) }\end{array}$ & $\begin{array}{l}\text { EC1: beet juice } 70 \mathrm{~mL} \\
\text { ( } 7 \text { mmol nitrate) }(150 \mathrm{~min} \\
\text { before) }\end{array}$ & $\begin{array}{l}\text { Incremental test in hypoxia } \\
(4000 \mathrm{~m}) \text {. Test of } 10 \mathrm{~km} \text { in hypoxia } \\
(2500 \mathrm{~m})\end{array}$ & No differences between variables \\
\hline [42] & $\begin{array}{l}\text { M, trained } \\
\text { athletes }(n: 10)\end{array}$ & $\begin{array}{l}\text { EC1: acute beet juice, } \\
\text { EC2: acute placebo, } \\
\text { EC1: chronic beet juice, } \\
\text { EC2: chronic placebo }\end{array}$ & $\begin{array}{l}\text { EC1: } 210 \mathrm{~mL} \text { beet juice } \\
\text { ( } 6.5 \mathrm{mmol} \text { nitrate) (150 } \\
\text { min before), EC2: placebo } \\
\text { (150 min before), EC1: } 210 \\
\text { mL beet juice ( } 6.5 \mathrm{mmol} \\
\text { nitrate) ( } 8 \text { days), } \\
\text { EC2: placebo ( } 8 \text { days) }\end{array}$ & $\begin{array}{l}19 \min \text { test }\left(7 \mathrm{~min} 50 \% \mathrm{VO}_{2 \max }+\right. \\
7 \mathrm{~min} \text { at } 65 \% \mathrm{VO}_{2 \max }+5 \mathrm{~min} \text { at } \\
\left.80 \% \mathrm{VO}_{2 \max }\right)+ \text { test of } 1500 \mathrm{~m}: \\
\text { respiratory parameters (test } \\
19 \text { min), performance (test } 1500 \mathrm{~m})\end{array}$ & $\begin{array}{l}\text { No significant differences between experimental } \\
\text { conditions }\end{array}$ \\
\hline$[43]^{*}$ & $\begin{array}{l}\text { M, trained } \\
\text { athletes }(n: 12)\end{array}$ & $\begin{array}{l}\text { EC1: normoxia + beet } \\
\text { juice, EC2: normoxia } \\
\text { placebo, EC3: hypoxia } \\
(2500 \mathrm{~m})+\text { beet juice, } \\
\text { EC4: hypoxia }(2500 \mathrm{~m})\end{array}$ & $\begin{array}{l}\text { EC1: beet juice } 140 \mathrm{~mL} \\
(8.4 \text { mmol nitrate) ( } 3 \text { days), } \\
\text { EC3: beet juice } 140 \mathrm{~mL} \\
\text { ( } 8.4 \text { mmol nitrate) ( } 3 \text { days) }\end{array}$ & $\begin{array}{l}\text { Test } 5 \text { min to } 80 \% \text { VT1 }+5 \text { min to } \\
75 \% \text { between } \mathrm{VT} 1 \text { and } \mathrm{VO}_{2 \max }+ \\
\text { time to exhaustion at } 75 \% \text { between } \\
\text { VT1 and } \mathrm{VO}_{2 \max } \text { : respiratory } \\
\text { parameters, performance, HR }\end{array}$ & $\begin{array}{l}\text { Respiratory parameters ( } 5 \text { min at } 80 \% \text { VT1): } \mathrm{VO}_{2}: \text { lower } \\
\text { in EC } 3 \text { vs. EC } 4 \text {, performance (time to exhaustion at } 75 \% \\
\left.\text { between VT1 and } \mathrm{VO}_{2 \max }\right): \text { higher in EC3 vs. EC } 4 \\
(214 \pm 14 \text { vs. } 197 \pm 28 \mathrm{~s})\end{array}$ \\
\hline$[44]^{*}$ & $\begin{array}{l}\text { M, trained } \\
\text { athletes }(n: 15)\end{array}$ & $\begin{array}{l}\text { EC1: normoxia + chronic } \\
\text { beet juice, EC2: normoxia } \\
\text { + placebo, EC3: hypoxia } \\
(5000 \mathrm{~m})+\text { chronic beet } \\
\text { juice, EC4: hypoxia } \\
(5000 \mathrm{~m})+\text { placebo }\end{array}$ & $\begin{array}{l}\text { EC1: beet juice } 500 \mathrm{~mL} \\
(0.7 \mathrm{mmol} \text { nitrate } / \mathrm{kg}) \\
(6 \text { days), } \mathrm{EC} 3: 70 \mathrm{~mL} \text { beet } \\
\text { juice }(0.7 \mathrm{mmol} \\
\text { nitrate } / \mathrm{kg})(6 \text { days })\end{array}$ & $\begin{array}{l}\text { Test } 20 \text { min at } 45 \% \mathrm{VO}_{2 \max }+ \\
\text { incremental test: respiratory } \\
\text { parameters, lactate, performance, } \\
\mathrm{HR}, \mathrm{RPE}\end{array}$ & $\begin{array}{l}\text { Test } 20 \mathrm{~min} \text { at } 45 \% \mathrm{VO}_{2 \mathrm{max}}: \mathrm{VO}_{2} \text { : lower in } \mathrm{EC} 3 \text { vs. } \mathrm{EC} 4 \text { at } \\
\text { rest }(8 \%) \text { and exercise }(4 \%), \mathrm{Incremental} \text { test: time to } \\
\text { exhaustion: higher EC2 vs. EC4 ( }(527 \pm 22 \mathrm{vs} .568 \pm 23 \mathrm{~s}) \text {, } \\
\text { Max. lactate: lower in EC1 vs. EC2 }(9.1 \pm 0.5 \mathrm{vs} \text {. } \\
10.6 \pm 0.3 \mathrm{mmol} / \mathrm{L})\end{array}$ \\
\hline [45] & $\begin{array}{l}\text { M, trained } \\
\text { athletes }(n: 14)\end{array}$ & $\begin{array}{l}\text { EC1: beet juice + caffeine, } \\
\text { EC2: caffeine + placebo, } \\
\text { EC3: beet juice + placebo, } \\
\text { EC4: placebo }\end{array}$ & $\begin{array}{l}\text { EC1: } 140 \mathrm{~mL} \text { beet juice } \\
(8 \mathrm{mmol} \text { nitrate })(90 \mathrm{~min} \\
\text { before })+5 \mathrm{mg} \cdot \mathrm{kg}^{-1} \text { of } \\
\text { caffeine }(60 \mathrm{~min} \text { before }) \text {, } \\
\text { EC2: } 5 \mathrm{mg} \cdot \mathrm{kg}^{-1} \text { of } \\
\text { caffeine }(40 \mathrm{~min} \text { before }), \\
\text { EC } 3: 2 \times 70 \mathrm{~mL} \text { beet juice } \\
(8 \mathrm{mmol} \text { nitrate }) \text { ( } 90 \mathrm{~min} \\
\text { before) }\end{array}$ & $\begin{array}{l}\text { Test } 30 \text { min } 60 \%+\text { test to } \\
\text { exhaustion at } 80 \% \mathrm{VO}_{2 \max } \text { : } \\
\text { respiratory parameters, } \\
\text { performance (test to exhaustion at } \\
\left.80 \% \mathrm{VO}_{2 \max }\right), \mathrm{HR}, \mathrm{RPE} \text {, cortisol }\end{array}$ & $\begin{array}{l}\text { RPE: lower at } 15 \text { min in test to exhaustion at } 80 \% \mathrm{VO}_{2 \max } \\
\text { in EC1 }(17 \pm 1) \text { vs. EC2 }(18 \pm 1) \text { and } \mathrm{EC} 4(19 \pm 2)\end{array}$ \\
\hline [46] & $\begin{array}{l}\text { W, trained } \\
\text { cyclists and } \\
\text { triathletes }(n: 14)\end{array}$ & $\begin{array}{l}\text { EC1: beet juice + caffeine, } \\
\text { EC2: caffeine + placebo, } \\
\text { EC3: beet juice + placebo, } \\
\text { EC4: placebo }\end{array}$ & $\begin{array}{l}\text { EC1: beet juice } 70 \mathrm{~mL} \\
\text { ( } 7.3 \mathrm{mmol} \text { nitrate) }(150 \\
\text { min before })+5 \mathrm{mg} \cdot \mathrm{kg}^{-1} \\
\text { of caffeine }(60 \mathrm{~min} \text { before), } \\
\text { EC2: } 5 \mathrm{mg} \cdot \mathrm{kg}^{-1} \text { of } \\
\text { caffeine }(60 \mathrm{~min} \text { before), } \\
\text { EC3: beet juice } 70 \mathrm{~mL} \\
\text { ( } 7.3 \mathrm{mmol} \text { nitrate) } \\
\text { ( } 150 \text { min before) }\end{array}$ & $\begin{array}{l}\text { Test of } 20 \mathrm{~km} \text { : respiratory } \\
\text { parameters, lactate, performance, } \\
\text { HR, RPE }\end{array}$ & $\begin{array}{l}\text { Respiratory parameters: RER: EC2 vs. EC3 }(+0.034) \text { and } \\
\text { EC4 }(+0.033) \text {, lactate: EC2 vs. EC3 }(+2.28 \mathrm{mmol} / \mathrm{L}) \text { and } \\
\text { EC4 }(+2.04 \mathrm{mmol} / \mathrm{L}) \text { and EC1 vs. EC3 }(+2.74 \mathrm{mmol} / \mathrm{L}) \\
\text { and EC4 }(+2.50 \mathrm{mmol} / \mathrm{L}), \text { Performance: power: EC2 vs. } \\
\text { EC3 improvement }(+10.3 \mathrm{~W}) \text { and EC4 }(+10.4 \mathrm{~W}) \text {, time: } \\
\text { improved EC2 vs. EC3 }(+42.4 \mathrm{~s}) \text { and EC3 }(+45.1 \mathrm{~s}), \mathrm{HR} \text { : } \\
\text { EC1 vs. EC2 vs. }(+8.0 \mathrm{bpm}), \mathrm{EC} 3(+5.2 \mathrm{bpm}) \text { and } \\
\text { EC4 }(+6.5 \mathrm{bpm})\end{array}$ \\
\hline
\end{tabular}


Table 2. Cont.

\begin{tabular}{|c|c|c|c|c|c|}
\hline Reference & Participants & Experimental Conditions & $\begin{array}{c}\text { Supplementation } \\
\text { Protocol }\end{array}$ & Variables & Results \\
\hline [47] & $\begin{array}{l}\text { M }(n: 12) \text { and } \\
\mathrm{W}(n: 12) \text {, trained } \\
\text { cyclists-triathletes } \\
(n: 24)\end{array}$ & $\begin{array}{l}\text { EC1: beet juice + caffeine, } \\
\text { EC2: caffeine + placebo, } \\
\text { EC3: beet juice + placebo, } \\
\text { EC4: placebo }\end{array}$ & $\begin{array}{l}\text { EC1: } 140 \mathrm{~mL} \text { beet juice } \\
(8.4 \mathrm{mmol} \text { nitrate })(8-12 \mathrm{~h} \\
\text { before })+3 \mathrm{mg} \cdot \mathrm{kg}^{-1} \text { of } \\
\text { caffeine }(60 \mathrm{~min} \text { before }), \\
\text { EC2: } 3 \mathrm{mg} \cdot \mathrm{kg}^{-1} \text { of } \\
\text { caffeine }(40 \mathrm{~min} \text { before }) \text {, } \\
\text { EC3: } 140 \mathrm{~mL} \text { beet juice } \\
\text { ( } 8.4 \mathrm{mmol} \text { nitrate })(8-12 \mathrm{~h} \\
\text { before) }\end{array}$ & $\begin{array}{l}\text { Test of } 43.83 \mathrm{~km} \mathrm{M} \text { and } 29.35 \mathrm{~km} \mathrm{~W} \text { : } \\
\text { performance, HR, RPE }\end{array}$ & $\begin{array}{l}\text { Performance: power: improvement in EC1 }(258 \pm 59 \text { W) } \\
\text { and EC2 (260 } \pm 58 \text { W) vs. EC4 ( } 250 \pm 57 \text { W), M time: } \\
\text { improvement EC1 (1:02:38 } \pm 0: 03: 31 \text { h:min:s) and EC2 } \\
\text { (1:02:43 } \pm 0: 03: 04 \text { h:min:s) vs. EC4 (1:03: } 30 \pm 0: 03: 16 \\
\text { h:min:s), W time: improving EC1 (0: } 51: 1 \pm 0: 02: 22 \\
\text { h:min:s) and EC2 (0:50:50 } \pm \text { 0:02:56 h:min:s) vs. EC4 (0:51: } \\
40 \pm 0: 02: 31 \text { h:min:s) in W }\end{array}$ \\
\hline [48] & $\begin{array}{l}\mathrm{M} \text {, trained } \\
\text { athletes }(n: 22)\end{array}$ & $\begin{array}{l}\text { EC1: Beet juice }(n: 11) \text {, } \\
\text { EC2: placebo }(n: 11)\end{array}$ & $\begin{array}{l}6 \text { weeks: EC1: } 500 \mathrm{~mL} \\
\text { beet juice ( } 5.8 \mathrm{mmol} \\
\text { nitrate, approximately) + } \\
\text { training in hypoxia } \\
(4000 \mathrm{~m}), \mathrm{EC} 2: \text { placebo + } \\
\text { hypoxia training }(4000 \mathrm{~m})\end{array}$ & $\begin{array}{l}\text { Progressive incremental test } 30 \mathrm{~min} \\
\text { test: respiratory parameters, lactate, } \\
\text { muscle glycogen, performance, HR }\end{array}$ & 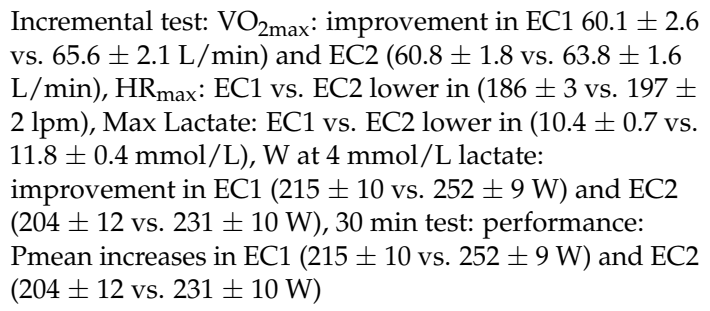 \\
\hline [49] & $\begin{array}{l}\text { M, trained } \\
\text { athletes }(n: 8)\end{array}$ & $\begin{array}{l}\text { EC1: beet juice, } \\
\text { EC2: beet juice }+ \\
\text { Mouthwash with } \\
\text { carbohydrates, } \\
\text { EC3: placebo }\end{array}$ & $\begin{array}{l}\text { EC1: } 140 \mathrm{~mL} \text { of beet juice } \\
\text { ( } 8 \mathrm{mmol} \text { nitrate) }(150 \mathrm{~min} \\
\text { before), EC2: beet juice } \\
140 \mathrm{~mL} \text { ( } 8 \mathrm{mmol} \text { nitrate })+ \\
\text { mouthwash } \\
\text { carbohydrates ( } 150 \mathrm{~min} \\
\text { before) }\end{array}$ & $\begin{array}{l}\text { Test } 60 \text { min at } 65 \% \mathrm{VO}_{2 \max } \text { : } \\
\text { respiratory parameters, lactate, } \\
\text { glucose, insulin, muscle glycogen, } \\
\text { ATP, creatine }\end{array}$ & $\begin{array}{l}\text { Lactate: increased EC1, EC2 and EC3. No differences } \\
\text { between groups. Muscle glycogen: decline in EC1, EC2 } \\
\text { and EC3. No differences between groups Creatine decline } \\
\text { in EC1, EC2 and EC3. No differences between groups }\end{array}$ \\
\hline
\end{tabular}

ATP: adenosine triphosphate; EC: experimental condition; HR: heart rate; M: men; h: hours; kg: kilograms; km: kilometers; bpm: beats per minute; m: meter; min: minutes; mL: milliliter; MAP: maximal aerobic power; RER: respiratory exchange rate; RPE: subjective perception of effort; s: sec; VT1: first ventilatory threshold; VO $\mathrm{V}_{2}$ : oxygen consumption; $\mathrm{VO}_{2 \text { max }}$ : maximal oxygen consumption; W: Watt. All results presented reflect statistically significant differences $(p<0.05)$. ${ }^{*}$ Studies where only the effect of beet juice vs. placebo in both hypoxic situations as compared normoxic condition. 
In Table 2, 23 articles were examined regarding beetroot juice supplementation in normoxic conditions, hypoxic conditions and beetroot juice combined with caffeine supplementation: 11 of those articles were related to trained athletes, four of them to cyclists-triathletes, three to cyclists trained, two to trained kayakers, one to trained runners, one to trained swimmers, and one to healthy physically active people. Twenty-one of these articles assessed respiratory parameters including $\mathrm{VO}_{2}$ at several intensities (approximately $60 \%-100 \% \mathrm{VO}_{2 \max }, \mathrm{VT} 1$ )

Briefly, in trained athletes men and women in normoxia conditions appeared that beetroot juice supplementation enhances aerobic performance by a decrease in $\mathrm{VO}_{2}$ at several intensities $(60 \%-100 \%$ $\mathrm{VO}_{2 \max }, \mathrm{VT1}$ ) increasing the economy during exercise. In kayak studies, a decrease of $\mathrm{VO}_{2}$ at the same intensity in kayakers supplemented with beetroot juice compared to a placebo group was found. In trained swimmers, a decrease in energy expenditure in the experimental condition of beetroot juice supplementation was observed.

Regarding the supplementation with beetroot juice in hypoxic conditions, five studies were selected. The hypothesis that beetroot improves cardiorespiratory performance in hypoxic conditions is controversial.

Two studies evaluated the effect of the combination of beetroot juice and caffeine in men and women trained cyclists-triathletes, and one study evaluated the same supplementation in trained men athletes. The studies did not determine that the effects of beetroot juice combined with caffeine increase the cardiorespiratory performance regarding caffeine supplementation.

\subsection{Acute Effects of Beetroot Juice Supplementation on Performance in Cardiorespiratory Endurance}

Several studies have shown a positive effect of acute beetroot juice intake on various parameters of performance improvement associated with the cardiovascular and respiratory system. Economy is a parameter that expresses the relationship between oxygen consumption $\left(\mathrm{VO}_{2}\right)$ and power generated or the distance traveled by an athlete [29], regarded as a performance factor in cardiorespiratory endurance [3-5]. Improved economy is due to achieving higher output power with the same $\mathrm{VO}_{2}$ level [30]. Another improvement attributed to beetroot juice supplementation is related to the increased blood flow, favoring the supply of oxygen to the mitochondria [50], which has the side effect of stimulating oxidative metabolism. In addition, supplementation with $\mathrm{NO}_{3}{ }^{-}$could improve the processes of muscle contraction and relaxation [31].

A study in trained cyclists found that beetroot juice supplementation improves performance by $0.8 \%$ in a 50 -mile test [32]. Significant increases in efficiency, measured as watts (W) per liter of $\mathrm{VO}_{2}$ $\left(\mathrm{W} / \mathrm{VO}_{2}\right)$ were observed in the last 10 miles; these improvements were associated with a decrease in time required to travel this distance. Another study [33] aimed to assess efficiency on a 40-min test at submaximal intensity ( $20 \mathrm{~min}$ at $50 \% \mathrm{VO}_{2 \max }$ followed by $20 \mathrm{~min}$ at $70 \% \mathrm{VO}_{2 \max }$ ). A decrease in $\mathrm{VO}_{2}$ and improved efficiency was also observed after beetroot juice supplementation, but did not reach statistical significance. After supplementation and immediately after the submaximal 40-min test, the time-to-exhaustion at an intensity of $90 \% \mathrm{VO}_{2 \max }$ improved as much as $16 \%$ in the trained cyclists. These findings make us suspect that beetroot juice might have an ergogenic effect, increasing performance in prolonged cycling events that require alternations in relative intensity, from moderate to high $\mathrm{VO}_{2 \max }$, which is very characteristic of the stages of cycling races.

In a time trial of $16.1 \mathrm{~km}$, supplementation with beetroot juice improved the performance of trained cyclists diminishing a completion time in a $2.7 \%$ and by $2.8 \%$ in a $4-\mathrm{km}$ time trial [27]. Although, the protocol test used in this study had a high ecological validity, providing an accurate simulation of the physiological responses during competition, it is unclear that beetroot supplementation can increase the performance by this magnitude in elite cyclist [27].

This increased performance was also associated with $\mathrm{W} / \mathrm{VO}_{2}$ improvements of $7 \%$ in a time trial of $16.1 \mathrm{~km}$ and $11 \%$ in $4-\mathrm{km}$ time trial [27]. The observed improvements in efficiency match those found in high-performance kayakers when paddling at $60 \%$ relative $\mathrm{VO}_{2 \max }$ intensity or in a 4-min test [34]. 
Response to a submaximal $\mathrm{VO}_{2}$ test at constant load is very important to cardiorespiratory endurance in athletic performance. In this type of test, $\mathrm{VO}_{2}$ increases disproportionately during the first $3 \mathrm{~min}$ because of an increase at the respiratory center to meet the exercise-induced increase in energy demand [51]. At an intensity below VT1 $60 \% \mathrm{VO}_{2 \max }$ efforts, approximately stabilization of $\mathrm{VO}_{2}$ is observed from the 3-min point until the end of the effort [52]. Nonetheless, at intensities greater than VT1 a progressively greater recruitment of type II motor units occurs [53], which have a lower oxidative potential than type I [54], and therefore a progressive increase in $\mathrm{VO}_{2}$ is observed from the third minute until the end of the exercise. This has been called the slow component of $\mathrm{VO}_{2}$ [55], which has been identified as one of the main factors limiting performance in endurance exercise of moderate and/or high intensity [4], because the increase in the slow component of $\mathrm{VO}_{2}$ attains values of $\mathrm{VO}_{2} \max$ at submaximal intensity, causing fatigue [56].

In experienced athletes, the effect of supplementation with beetroot juice ( $8.2 \mathrm{mmol}$ nitrate) on time-to-exhaustion was tested at intensities of $60 \%, 70 \%, 80 \%$ and $100 \%$ peak power [35]. Athletes were able to maintain an intensity of $60 \%$ (Beetroot: $696 \pm 120$ vs. Placebo: $593 \pm 68$ s), 70\% (Beetroot: $452 \pm 106$ vs. Placebo: $390 \pm 86$ s) and 80\% (Beetroot: $294 \pm 50$ vs. Placebo: $263 \pm 50$ s) peak power significantly longer during exercise with supplementation, and there was a trend toward increased endurance at $100 \%$ peak power. The study results might reflect a lower $\mathrm{VO}_{2}$ response at submaximal intensities, which would reduce the increase in the slow component, delaying the time when the athletes reached $\mathrm{VO}_{2 \max }$ and therefore became fatigued. This would allow a longer sustained effort.

On the other hand, trained runners participating in a 5000-m test showed no significant overall improvement with beetroot juice supplementation, although they ran $5 \%$ faster in the later part of the race, particularly the last 1.1 miles [12]. The lack of significance could be related to the timing of the supplementation. Participants took the supplement $90 \mathrm{~min}$ before exercise; in the other studies cited, beetroot juice was provided 150-180 min before the effort [27,32-35] and ergogenic effects of supplementation with beetroot juice were observed at $150 \mathrm{~min}$ after ingestion [35].

\subsection{Effects of Chronic Supplementation with Beetroot Juice on Cardiorespiratory Endurance}

In addition to increasing blood flow and improving muscle contraction and relaxation, beetroot juice supplementation may improve the efficiency of mitochondrial respiration [50] and oxidative phosphorylation [57]. It seems, however, that acute supplementation is insufficient to produce mitochondrial biogenesis, suggesting that these adaptations may require longer supplementation protocols. In trained athletes, acute supplementation with beetroot juice for five days reduces $\mathrm{VO}_{2}$ as much as $3 \%$ at an intensity of $70 \% \mathrm{VO}_{2 \max }$. The test was performed at $50 \% \mathrm{VO}_{2 \max }$ for $10 \mathrm{~min}$, followed by $10 \mathrm{~min}$ at $70 \% \mathrm{VO}_{2 \max }$ [31]. Another study in trained cyclists confirmed that supplementation for a period of six days reduces $\mathrm{VO}_{2}$ in a 60 -min test. The protocol consisted of $30 \mathrm{~min}$ at $45 \% \mathrm{VO}_{2} \max$ followed by another $30 \mathrm{~min}$ at $65 \% \mathrm{VO}_{2 \max }$. In addition, riders were able to improve their $10-\mathrm{km}$ time trial performance immediately following the submaximal test [30].

These studies clarify the benefits that could result from supplementation with beetroot juice in longer intake protocols of about six days, as was the case in the time-to-exhaustion test at submaximal intensities following acute supplementation $[33,35]$. Time-to-exhaustion improved at intensities of $70 \%$ of $\mathrm{VO}_{2 \max }$, between $\mathrm{VT} 1$ and $\mathrm{VO}_{2 \max }$ [37]. In trained swimmers, Pinna et al. [38] also corroborated the progressive ergogenic benefits of beetroot juice during an incremental test. At anaerobic threshold intensity, workload increased and aerobic energy expenditure decreased.

In another study, in healthy subjects physically active but not highly trained in any particular sport, Vanhatalo et al. [36] evaluated the acute and chronic (15-day) effects of dietary supplementation with $\mathrm{NO}_{3}{ }^{-}$on $\mathrm{VO}_{2}$ in a constant load test at an intensity of $90 \%$ of the gas exchange threshold (GET), similar to the anaerobic threshold, and in a progressive incremental ergometric cycle test, compared to controls. The peak power in the incremental test and the ratio of work rate to GET intensity were increased in the group that received the dietary $\mathrm{NO}_{3}{ }^{-}$supplementation. The findings indicated that 
dietary supplementation reduces $\mathrm{NO}_{3}{ }^{-}$oxygen consumption at submaximal exercise, and these effects can last for 15 days if supplementation is maintained.

Potential improvements observed in the anaerobic or lactate threshold intensity is especially important for athletes in various forms of endurance sports, because the level achieved in this parameter does not depend on motivation as it occurs when $\mathrm{VO}_{2 \max }$ is determined [58]. This threshold is considered a factor that better discriminates between cardiorespiratory endurance capacities than does $\mathrm{VO}_{2 \max }[2,58]$. One of the physiological parameters that conditions improvement in the anaerobic threshold is increased mitochondrial population [59]. If the beetroot juice supplementation can promote mitochondrial biogenesis, we might assume that chronic supplementation with beetroot juice would decrease oxygen consumption at anaerobic threshold intensity as an adaptation to exercise.

It has also been suggested that additional beetroot juice supplementation may improve the muscle contraction functions. A study by Whitfield et al. [31] found that $\mathrm{VO}_{2}$ reduction after a constant load test at $70 \% \mathrm{VO}_{2 \max }$ occurred without any changes in markers of mitochondrial efficiency such as adenine nucleotide translocase (ANT) and uncoupling protein 3 (UCP3). Similarly, other researchers have suggested that supplementation may positively affect the interaction of actin and myosin bridges [60] by modulating the release of calcium that occurs after the action potential [61]. The effects described by these authors indicate that supplementation with beetroot juice, whether acute or chronic, could improve performance in sports that are characterized either by a predominantly aerobic or anaerobic metabolism [38]. This could explain the positive effects on effort with a high prevalence of anaerobic metabolism observed in a 500-m kayak test [29] or in the contractile force developed by mice [62].

\subsection{Effects of Beetroot Juice Supplementation on Performance in Cardiorespiratory Endurance under Hypoxic Conditions}

Many competitions, such as the mountain stages in cycling, are held at high altitudes [39], where cardiorespiratory endurance is decreased relative to sea level [63]. Among the factors that could be responsible for this decrease, we would highlight decreased supply of oxygen to muscles, due to a partial reduction in oxygen pressure.

It is known that $\mathrm{NO}$ has an important role in the adaptation processes under hypoxic conditions; higher levels of $\mathrm{NO}_{2}{ }^{-}$have been observed in Tibetans [18]. In a study of acute response to hypoxia, people who live at sea level who climb to high altitudes and show decreased NO levels have symptoms of acute altitude sickness [64,65]. The vasodilatory effects of NO may favor oxygen delivery [66], and supplementation with beetroot juice could be effective in reducing the ergolytic effects of hypoxia on cardiorespiratory endurance [39].

A recent study evaluated the effects of supplementation with acute and chronic beetroot juice on a 15-min test at an intensity of $50 \% \mathrm{VO}_{2 \max }$ and a $10-\mathrm{km}$ test carried out at a simulated altitude of $2500 \mathrm{~m}$ [40]. The test could not verify any positive effect of acute or chronic supplementation on any of the performance variables analyzed. In addition, studies have shown supplementation with beetroot juice did not improve performance in runners with a high level of training in an incremental intensity test or in a 10-km race [41] or in a $1500-\mathrm{m}$ test or tests at various submaximal intensities $(50 \%, 65 \%$ and $80 \% \mathrm{VO}_{2 \max }$ ) [42]. The results in the latter study are also in line with those reported by McLeod [40]; in these two studies, beetroot juice was administered 90 and $120 \mathrm{~min}$, respectively, before exercise. This may be an insufficient time interval for athletes to reach peak $\mathrm{NO}_{2}{ }^{-}$levels in their bloodstream.

The results presented above conflict with other reports [39,43]. Kelly et al. [43] tested the effect of beetroot juice supplementation for three days on performance in a 5-min test at $80 \%$ VT1, followed by a test to the point of exhaustion at an intensity at $75 \%$ of $\mathrm{VT} 1$ and $\mathrm{VO}_{2 \max }$ and a simulated altitude of $2500 \mathrm{~m}$. The results show that supplementation with beetroot juice reduced $\mathrm{VO}_{2}$ to $80 \% \mathrm{VT} 1$ and there was a statistical trend to improvement in higher intensity exercise $(p=0.07)$. Improved efficiency was accompanied by a longer time-to-exhaustion in a test at $75 \%$ between VT1 and $\mathrm{VO}_{2 \max }$. In another study that simulated an altitude of $2500 \mathrm{~m}$, supplementation with beetroot juice again reduced the 
$\mathrm{VO}_{2}$ during a $15-\mathrm{min}$ test at $60 \%$ of $\mathrm{VO}_{2 \max }$ and increased the speed achieved in a 16.1- $\mathrm{km}$ time trial involving trained cyclists [39]. The results observed in the time trial were consistent with the improvements $(2.8 \%)$ reported from a $4-\mathrm{km}$ time trial after a protocol of acute supplementation with beetroot juice [27].

Masschelein et al. [44] found that six days of supplementation with beetroot juice can reduce $\mathrm{VO}_{2}$ at rest by $8 \%$, and by $4 \%$ at $45 \% \mathrm{VO}_{2 \max }$ intensity at a simulated altitude of $5000 \mathrm{~m}$. Although the cited study is not directly generalizable to performance in various types of cardiorespiratory endurance, as competitions are unlikely to take place above an altitude of $2500 \mathrm{~m}$, other parameters such as arterial oxygen saturation $\left(\mathrm{SPO}_{2}\right)$ and deoxyhemoglobin $(\mathrm{HHb})$ in muscle tissue were analyzed. The results showed that reductions in $\mathrm{VO}_{2}$ were accompanied by greater $\mathrm{SPO}_{2}$ and lower $\mathrm{HHb}$ after supplementation with beetroot juice, indicating decreased oxygen extraction by the muscle, which coincides with increased mechanical pedaling efficiency and lower levels of lactate in the blood.

Although the literature shows contradictory data, it is possible that supplementation with beetroot juice may effectively improve performance when hypoxia is present, because oxygenation would improve at the muscular level, reducing the ergolytic effects of hypoxia on aerobic performance.

\subsection{Effects of the Combination of Beetroot Juice Supplementation with Other Supplements on Cardiorespiratory Endurance}

Caffeine supplementation has become increasingly common among athletes [67]. Among its positive effects is increased stimulation of the central nervous system due to the antagonism of adenosine [68], increased catecholamines and contractility of skeletal muscle [69] that improves calcium output from the sarcoplasmic reticulum through the action potential [70], and a decrease in the subjective perception of pain and the regulation of thermoregulation [71]. Thus, caffeine supplementation has proven ergogenic effects on various modalities of cardiorespiratory endurance [72] and team sports [73,74]. A plateau effect occurs in performance improvement, at doses ranging from 3 to $6 \mathrm{mg} / \mathrm{kg}$ of caffeine [75]. To test whether the combined supplementation of beetroot juice $\left(8 \mathrm{mmol}\right.$ of $\left.\mathrm{NO}_{3}{ }^{-}\right)$and caffeine $(5 \mathrm{mg} / \mathrm{kg})$ had a greater effect than each supplement separately, researchers tested the corresponding study groups of cyclists tested for $30 \mathrm{~min}$ at $60 \% \mathrm{VO}_{2 \mathrm{max}}$, followed by a test to exhaustion at $80 \% \mathrm{VO}_{2 \max }$ [45]. Although the combined supplementation improved time to exhaustion $\mathrm{VO}_{2 \max } 80 \%$ by $46 \%$ compared to placebo, the improvement was insignificant. Furthermore, the additive effect of taking both supplements did not improve performance to a greater extent than separate supplementation with each one [46,47].

In a study that simulated the characteristics of an Olympic cycling time trial, the effect of supplementation in both men and women cyclists was tested using beetroot juice $(8.2 \mathrm{mmol}$ of $\left.\mathrm{NO}_{3}{ }^{-}\right)$and caffeine $(3 \mathrm{mg} / \mathrm{kg})$ and the combination of both [47]. The only proven effects were that caffeine supplementation in combination with beetroot juice was effective in improving mean power and time trial results.

In a later study of trained cyclists and triathletes, performance was improved only in the athletes who received a caffeine supplement $\left(3 \mathrm{mg} / \mathrm{kg}\right.$ ) [46]. No differences were observed in $\mathrm{VO}_{2}$. However, lactate concentration in the blood was increased when athletes received caffeine supplementation. Performance improvement was likely due to an increased anaerobic metabolism after caffeine intake; therefore, it is possible that the effects of supplementation with beetroot juice can be undermined by interaction with other supplements such as caffeine, which interferes with the effects of each supplement taken separately.

\subsection{Dosage}

Peak $\mathrm{NO}_{2}{ }^{-}$concentration in blood is obtained within 2-3 $\mathrm{h}$ of $\mathrm{NO}_{3}{ }^{-}$supplementation [76] and the ergogenic effects of supplementation with beetroot juice can be observed at 150 min after ingestion [36]. Oral antiseptic rinses should not be taken with beetroot juice supplementation, as these can prevent the desired increase in $\mathrm{NO}_{2}{ }^{-}$levels after $\mathrm{NO}_{3}{ }^{-}$ingestion [77]. Although the majority of studies show 
ergogenic effects of beetroot juice at a supplementation dose of $6-8 \mathrm{mmol} \mathrm{NO}_{3}{ }^{-}$(Table 2), it is possible that high performance athletes might require a slightly higher dose. For example, in high performance kayakers, the ergogenic effect of supplementation with beetroot juice was $1.7 \%$ in a $500-\mathrm{m}$ test after ingestion of $9.6 \mathrm{mmol}$ of $\mathrm{NO}_{3}{ }^{-}$but a $4.8 \mathrm{mmol}$ dose did not significantly improve results in a $1000-\mathrm{m}$ test [29].

\section{Practical Considerations}

It appears that acute supplementation with beetroot juice increases the power output with the same $\mathrm{VO}_{2}$ levels [30]. This is an interesting finding for athletes as there is evidence that the economy is a key factor to improve cardiorespiratory performance increasing energy efficiency in endurance sports modalities. In addition, time to exhaustion at several intensities $\left(60 \%-100 \% \mathrm{VO}_{2 \mathrm{max}}, \mathrm{MAP}\right.$ or VT1) is another usual performance parameter that is improved with acute beetroot supplementation $[33,35]$. However, not all studies show a positive effect to acute beetroot supplementation indicating that the efficacy of acute nitrate supplementation will be attributed to several factors such as the age, diet, physiological and training status, and other parameters as the intensity, duration, endurance modality and environment conditions [78]. Although most of the studies determine a supplementation dose of 6-8 $\mathrm{mmol} \mathrm{NO}_{3}{ }^{-}$, it is unclear that this supplementation dose can be effective to improve cardiorespiratory performance in sports modalities such as kayaking or rowing. The dose should possibly be increased in sports modalities where muscular groups of upper limbs are implicated. Endurance athletes should take the dose of $\mathrm{NO}_{3}{ }^{-}$, approximately 90 min before the competition without oral antiseptic. Acute supplementation with beetroot juice is not sufficient to induce mitochondrial biogenesis, suggesting that mitochondrial adaptations could only occur after longer supplementation protocols. In chronic supplementations with beetroot juice, it appears that the benefits in cardiorespiratory performance might be produced in longer intake protocols of about six days [33,35]. Time-to-exhaustion at several intensities (between $70 \%$ and $100 \% \mathrm{VO}_{2 m a x}, \mathrm{VT} 1$ ) and the load at anaerobic threshold could be enhanced while aerobic energy expenditure could be diminished. Longer-term beetroot supplementation (15 or more days) could be effective, although it would be necessary other studies analyzing the mitochondrial biogenesis to corroborate whether mitochondrial adaptations depend on endurance training and/or beetroot supplementation. To date, this assumption is unknown.

The scientific literature shows discrepancies regarding the improvement of the cardiorespiratory performance induced by the supplementation of beetroot juice under hypoxic conditions. $\mathrm{NO}_{3}{ }^{-} \mathrm{Could}^{-}$ mitigate the ergolytic effects of hypoxia on cardiorespiratory in endurance athletes [39].

We cannot assert that the combination of beetroot juice with other supplements has a positive or negative effect on cardiorespiratory endurance. It is possible that the effects of supplementation with beetroot juice can be undermined by interaction with other supplements such as caffeine. More work is needed to confirm the results of these investigations.

\section{Conclusions}

- $\quad$ Acute supplementation with beetroot juice may have an ergogenic effect on reducing $\mathrm{VO}_{2}$ at less than or equal to $\mathrm{VO}_{2 \mathrm{max}}$ intensity, while improving the relationship between watts required and $\mathrm{VO}_{2}$ level, mechanisms that make it possible to enable increase time-to-exhaustion at less than or equal to $\mathrm{VO}_{2 \max }$ intensity.

- In addition to improving efficiency and performance in various time trials or increasing time-to-exhaustion at submaximal intensities, chronic supplementation with beetroot juice may improve cardiorespiratory performance at the anaerobic threshold and $\mathrm{VO}_{2 m a x}$ intensities. 
- Apparently, the effects of supplementation with beetroot juice might not have a positive interaction with caffeine supplementation, mitigating the effects of beetroot juice intake on cardiorespiratory performance, however, more work is needed to confirm the results of these investigations because the number of studies analyzing the effects of the combination of beetroot juice with other supplements, such as caffeine, is limited.

- Intake of beetroot juice should be initiated within $90 \mathrm{~min}$ before athletic effort, since the peak value of $\mathrm{NO}_{3}{ }^{-}$occurs within 2-3 h after ingestion. At least 6-8 mmol of $\mathrm{NO}_{3}{ }^{-}$intake is required, which can be increased in athletes with a high level of training.

Author Contributions: R.D. and M.V.G.-C. conception and design of review; R.D., E.C., J.L.M.-M., P.G.-F., M.C.L.E., P.V.H. and M.V.G.-C. carried out the bibliographic review; R.D. and M.V.G.-C. carried out the figures, tables and drafted manuscript; N.S.-P. and M.V.G.-C. performed the English translation; R.D., E.C., J.L.M.-M., P.G.-F., N.S.-P., M.C.L.E., P.V.H. and M.V.G.-C. edited and revised manuscript; R.D., E.C., J.L.M.-M., P.G.-F., N.S.-P., M.C.L.E., P.V.H. and M.V.G.-C. approved final version of manuscript.

Conflicts of Interest: The authors declare no conflict of interest.

\section{References}

1. Caspersen, C.J.; Powell, K.E.; Christenson, G.M. Physical activity, exercise, and physical fitness: Definitions and distinctions for health-related research. Public Health Rep. 1985, 100, 126-131. [PubMed]

2. Bassett, D.R.; Howley, E.T. Limiting factors for máximum oxygen uptake and determinants of endurance performance. Med. Sci. Sports Exerc. 2000, 32, 70-84. [CrossRef] [PubMed]

3. Bentley, D.J.; Newell, J.; Bishop, D. Incremental Exercise Test Design and Analysis: Implications for Performance Diagnostics in Endurance Athletes. Sports Med. 2007, 37, 575-586. [CrossRef] [PubMed]

4. Burnley, B.; Jones, A.M. Oxygen uptake kinetics as a determinant of sports performance. Eur. J. Sport. Sci. 2007, 7, 63-79. [CrossRef]

5. Jung, A.P. The Impact of Resistance Training on Distance Running Performance. Sports Med. 2003, 33, 539-552. [CrossRef] [PubMed]

6. Paton, C.D.; Hopkins, W.G. Performance Enhancement at the Fifth World Congress on Sport Sciences; University of Otago: Dunedin, New Zealand, 1999; Volume 3.

7. Knapik, J.J.; Steelman, R.A.; Hoedebecke, S.S.; Austin, K.G.; Farina, E.K.; Leberman, H.R. Prevalence of dietary supplement use by athletes: Systematic review and meta-analysis. Sports Med. 2016, 46, 103-123. [CrossRef] [PubMed]

8. Rodríguez, N.R.; Rodríguez, N.S.; Di Marc, N.M.; Langley, S. American College of Sports Medicine position stand. Nutrition and athletic performance. Med. Sci. Sports Exerc. 2009, 41, 709-731. [PubMed]

9. Australian Institute of Sport. ABCD Classification System. 2016. Available online: http://www.ausport.gov. $\mathrm{au} /$ ais/nutrition/supplements/classification (accessed on 5 January 2017).

10. Close, G.L.; Hamilton, L.; Philps, A.; Burke, L.; Morton, J.P. New strategies in sport nutrition to increase Exercise Performance. Free Radic. Biol. Med. 2016, 98, 144-158. [CrossRef] [PubMed]

11. Burke, L. Nutrición en el Deporte; Medica Panamericana: Madrid, Spain, 2010.

12. Murphy, M.; Eliot, K.; Heuertz, R.; Weiss, E. Whole Beetroot Consumption Acutely Improves Running Performance. J. Acad. Nutr. Diet. 2012, 112, 548-552. [CrossRef] [PubMed]

13. Duncan, H.; Dougall, P.; Johnston, P.; Green, S.; Brogan, R.; Leifert, C.; Smith, L.; Golden, M.; Benjamin, N. Chemical generation of nitric oxide in the mouth from the enterosalivary circulation of dietary nitrate. Nat. Med. 1995, 1, 546-551. [CrossRef] [PubMed]

14. Lundberg, J.O.; Govoni, M. Inorganic nitrate is a possible source for systemic generation of nitric oxide. Free Radic. Biol. Med. 2004, 37, 395-400. [CrossRef] [PubMed]

15. Bailey, S.J.; Winyard, P.; Vanhatalo, A.; Blackwell, J.R.; Di Menna, F.J.; Wilkerson, D.P.; Tarr, J.; Benjamin, N.; Jones, A.M. Dietary nitrate supplementation reduces the $\mathrm{O}_{2}$ cost of low-intensity exercise and enhances tolerance to high-intensity exercise in humans. J. Appl. Physiol. 2009, 107, 1144-1155. [CrossRef] [PubMed]

16. Ferguson, S.K.; Hirai, D.M.; Copp, S.W.; Holdsworth, C.T.; Allen, J.D.; Jones, A.M.; Musch, T.I.; Poole, D.C. Impact of dietary nitrate supplementation via beetroot juice on exercising muscle vascular control in rats. J. Physiol. 2013, 591, 547-557. [CrossRef] [PubMed] 
17. Larsen, F.J.; Ekblom, B.; Lundberg, J.O.; Weitzberg, E. Effects of dietary nitrate on oxygen cost during exercise. Acta Physiol. 2007, 191, 59-66. [CrossRef] [PubMed]

18. Erzurum, S.C.; Ghosh, S.; Janocha, A.J.; Xu, W.; Bauer, S.; Bryan, N.S.; Tejero, J.; Hermann, C.; Hille, R.; Stuehr, D.J.; et al. Higher blood flow and circulating NO products offset high-altitude hypoxia among Tibetans. Proc. Natl. Acad. Sci. USA 2007, 104, 17593-17598. [CrossRef] [PubMed]

19. Stamler, J.S.; Meissner, G. Physiology of nitric oxide in skeletal muscle. Physiol. Rev. 2001, 81, $209-237$. [PubMed]

20. Andrade, F.H.; Reid, M.B.; Allen, D.G.; Westerblad, H. Effect of nitric oxide on single skeletal muscle fibres from the mouse. J. Physiol. 1998, 509, 577-586. [CrossRef] [PubMed]

21. Wink, D.A.; Hines, H.B.; Cheng, R.Y.; Switzer, C.H.; Flores-Santana, W.; Vitek, M.P.; Ridnour, L.A.; Colton, C.A. Nitric oxide and redox mechanisms in the immune response. J. Leukoc. Biol. 2011, 89, 873-891. [CrossRef] [PubMed]

22. Tong, L.; Heim, R.A.; Wu, S. Nitric oxide: A regulator of eukaryotic initiation factor 2 kinases. Free Radic. Biol. Med. 2011, 50, 1717-1725. [CrossRef] [PubMed]

23. Kerley, C.P.; Cahill, K.; Bolger, K.; McGowan, A.; Burke, C.; Faul, J.; Cromican, L. Dietary nitrate supplementation in COPD: An acute, double-blind, randomized, placebo-controlled, crossover trial. Nitric Oxide 2015, 44, 105-111. [CrossRef] [PubMed]

24. Kapil, V.; Khambata, R.S.; Robertson, A.; Caulfield, M.J.; Ahluwalia, A. Dietary nitrate provides sustained blood pressure lowering in hypertensive patients: A randomized, phase 2, double-blind, placebo-controlled study. Hypertension 2015, 65, 320-327. [CrossRef] [PubMed]

25. Zamani, P.; Rawat, D.; Shiva-Kumar, P.; Geraci, S.; Bhuva, R.; Konda, P.; Doulias, P.T.; Ischiropoulos, H.; Townsend, R.R.; Margulies, K.B.; et al. Effect of inorganic nitrate on exercise capacity in heart failure with preserved ejection fraction. Circulation 2015, 131, 371-380. [CrossRef] [PubMed]

26. Nyström, T.; Ortsäter, H.; Huang, Z.; Zhang, F.; Larsen, F.J.; Weitzberg, E.; Lundberg, J.O.; Sjöholm, Å. Inorganic nitrite stimulates pancreatic islet blood flow and insulin secretion. Free Radic. Biol. Med. 2012, 53, 1017-1023. [CrossRef] [PubMed]

27. Lansley, K.E.; Winyard, P.G.; Bailey, S.J.; Vanhatalo, A.; Wilkerson, D.P.; Blackwell, J.R.; Gilchrist, M.; Benjamin, N.; Jones, A.M. Acute Dietary Nitrate Supplementation Improves Cycling Time Trial Performance. Med. Sci. Sports Exerc. 2011, 43, 1125-1131. [CrossRef] [PubMed]

28. Berthon, P.; Fellman, N.; Bedu, M.; Beaune, B.; Dabonneville, M.; Coudert, J.; Chamouz, A. A 5-min running test as a mesaurement of maximal aerobic velocity. Eur. J. Appl. Physiol. Occup. Physiol. 1997, 75, 233-238. [CrossRef] [PubMed]

29. Peeling, P.; Cox, G.; Bullock, N.; Burke, L. Beetroot Juice Improves On-Water 500 M Time-Trial Performance, and Laboratory-Based Paddling Economy in National and International-Level Kayak Athletes. Int. J. Sport Nutr. Exerc. Metab. 2015, 25, 278-284. [CrossRef] [PubMed]

30. Cermak, N.; Gibala, M.; Van Loon, J. Nitrate Supplementation's Improvement of 10-km Time-Trial Performance in Trained Cyclists. Int. J. Sport Nutr. Exerc. Metab. 2012, 22, 64-71. [CrossRef] [PubMed]

31. Whitfield, J.; Ludzki, A.; Heigenhauser, G.; Senden, S.; Verdijk, L.; Van, L.; Spriet, L.L.; Holloway, G.P. Beetroot Juice Supplementation Reduces Whole Body Oxygen Consumption But Does Not Improve Indices Of Mitochondrial Efficiency in Human Skeletal Muscle. J. Physiol. 2016, 594, 421-435. [CrossRef]

32. Wilkerson, D.P.; Hayward, G.M.; Bailey, S.J.; Vanhatalo, A.; Blackwell, J.R.; Jones, A.M. Influence of acute dietary nitrate supplementation on 50 mile time trial performance in well-trained cyclists. Eur. J. Appl. Physiol. 2012, 112, 4127-4134. [CrossRef]

33. Thompson, K.; Turnerb, L.; Prichardb, J.; Doddb, F.; Kennedyb, D.; Haskellb, C.; Blackwell, J.R.; Jones, A.M. Influence of dietary nitrate supplementation on physiological and cognitive responses to incremental cycle exercise. Respir. Physiol. Neurobiol. 2014, 193, 11-20. [CrossRef] [PubMed]

34. Muggeridge, D.; Howe, D.; Spendiff, O.; Pedlar, C.; James, P.; Easton, C. The Effects of a Single Dose of Concentrated Beetroot Juice on Performance in Trained Flatwater Kayakers. Int. J. Sport Nutr. Exerc. Metab. 2013, 23, 498-506. [CrossRef] [PubMed]

35. Kelly, J.; Vanhatalo, A.; Wilkerson, D.; Wylie, L.; Jones, A.M. Effects of Nitrate on the Power-Duration Relationship for Severe-Intensity Exercise. Med. Sci. Sports Exerc. 2013, 45, 1798-1806. [CrossRef] [PubMed] 
36. Vanhatalo, A.; Bailey, S.J.; Blackwell, J.R.; DiMenna, F.J.; Pavey, T.G.; Wilkerson, D.P.; Benjamin, N.; Winyard, P.G.; Jones, A.M. Acute and chronic effects of dietary nitrate supplementation on blood pressure and the physiological responses to moderate-intensity and incremental exercise. Am. J. Physiol. Regul. Integr. Comp. Physiol. 2010, 299, 1121-1131. [CrossRef] [PubMed]

37. Breese, B.C.; McNarry, M.A.; Marwood, S.; Blackwell, J.R.; Bailey, S.J.; Jones, A.M. Beetroot juice supplementation speeds $\mathrm{O}_{2}$ uptake kinetics and improves exercise tolerance during severe-intensity exercise initiated from an elevated metabolic rate. Am. J. Physiol. Regul. Integr. Comp. Physiol. 2013, 305, 1441-1450. [CrossRef]

38. Pinna, M.; Roberto, S.; Milia, R.; Maronquiu, E.; Olla, S.; Loi, A.; Migliaccio, G.M.; Padulo, J.; Orlandi, C.; Tocco, F.; et al. Effect of beetroot juice supplementation on aerobic response during swimming. Nutrients 2014, 6, 605-615. [CrossRef] [PubMed]

39. Muggeridge, D.J.; Howe, C.; Spendiff, O.; Pedlar, C.; James, P.; Easton, C. A Single Dose of Beetroot Juice Enhances Cycling Performance in Simulated Altitude. Med. Sci. Sports Exerc. 2014, 46, 143-150. [CrossRef] [PubMed]

40. MacLeod, K.E.; Nugent, S.F.; Barr, S.; Khoele, M.S.; Sporer, B.C.; Maclnnis, M.J. Acute Beetroot Juice Supplementation Does Not Improve Cycling Performance in Normoxia or Moderate Hypoxia. Int. J. Sport Nutr. Exerc. Metab. 2015, 25, 359-366. [CrossRef] [PubMed]

41. Arnold, J.; James, L.; Jones, T.; Wylie, L.; Macdonald, J. Beetroot juice does not enhance altitude running performance in well-trained athletes. Appl. Physiol. Nutr. Metab. 2015, 40, 590-595. [CrossRef] [PubMed]

42. Boorsma, R.K.; Whitfield, S.L. Beetroot Juice Supplementation Does Not Improve Performance of Elite 1500-m Runners. Med. Sci. Sports Exerc. 2014, 46, 2326-2334. [CrossRef] [PubMed]

43. Kelly, J.; Vanhatalo, A.; Bailey, S.J.; Wylie, L.J.; Tucker, C.; List, S.; Winyard, P.G.; Jones, A.M. Dietary nitrate supplementation: Effects on plasma nitrite and pulmonary $\mathrm{O}_{2}$ uptake dynamics during exercise in hypoxia and normoxia. Am. J. Physiol. Regul. Integr. Comp. Physiol. 2014, 307, 920-930. [CrossRef] [PubMed]

44. Masschelein, E.; Van Thienen, R.; Wang, X.; Van Schepdael, A.; Thomis, M.; Hespel, P. Dietary nitrate improves muscle but not cerebral oxygenation status during exercise in hypoxia. J. Appl. Physiol. 2012, 113, 736-745. [CrossRef] [PubMed]

45. Handzlik, L.; Gleeson, M. Likely Additive Ergogenic Effects of Combined Preexercise Dietary Nitrate and Caffeine Ingestion in Trained Cyclists. ISRN Nutr. 2013, 2013, 396581. [CrossRef] [PubMed]

46. Glaister, M.; Pattison, J.R.; Muniz-Pumares, D.; Patterson, S.D.; Foley, P. Effects of dietary nitrate, caffeine, and their combination on 20-km cycling time trial performance. J. Strength Cond. Res. 2015, 29, 165-174. [CrossRef] [PubMed]

47. Lane, S.; Hawley, J.; Desbrow, B.; Jones, A.M.; Blackwell, J.; Ross, M.L.; Zemski, A.J.; Burke, L.M. Single and combined effects of beetroot juice and caffeine supplementation on cycling time trial performance. Appl. Physiol. Nutr. Metab. 2014, 39, 1050-1057. [CrossRef] [PubMed]

48. Puype, J.; Ramaekers, M.; Thienen, R.; Deldicque, L.; Hespel, P. No effect of dietary nitrate supplementation on endurance training in hipoxia. Scand. J. Med. Sci. Sports 2015, 25, 234-241. [CrossRef] [PubMed]

49. Betteridge, S.; Bescós, R.; Martorell, M.; Pons, A.; Garnham, A.P.; Stathis, C.C.; McConell, G.K. No effect of acute beetroot juice ingestion on oxygen consumption, glucose kinetics, or skeletal muscle metabolism during submaximal exercise in males. J. Appl. Physiol. 2016, 120, 391-398. [CrossRef] [PubMed]

50. Bailey, S.J.; Fulford, J.; Vanhatalo, A.; Winyard, P.G.; Blackwell, J.R.; DiMenna, F.J.; Wilkerson, D.P.; Benjamin, N.; Jones, A.M. Dietary nitrate supplementation enhances muscle contractile efficiency during knee-extensor exercise in humans. J. Appl. Physiol. 2010, 109, 135-148. [CrossRef] [PubMed]

51. Xu, F.; Rhodes, E.C. Oxygen uptake kinetics during exercise. Sports Med. 1999, 27, 313-327. [CrossRef] [PubMed]

52. Lucía, A.; Pardo, J.; Durántez, A.; Hoyos, J.; Chicharro, J.L. Physiological differences between professional and elite road cyclists. Int. J. Sports Med. 1998, 19, 342-348. [CrossRef] [PubMed]

53. Pérez, M.; Santalla, A.; Chicharro, J.L. Effects of electrical stimulation on $\mathrm{VO}_{2}$ kinetics and delta efficiency in healthy young men. Br. J. Sports Med. 2003, 37, 140-143. [CrossRef] [PubMed]

54. Lucía, A.; Sánchez, O.; Carvajal, A.; Chicharro, J.L. Analysis of the aerobic-anaerobic transition in elite cyclists during incremental exercise with the use of electromyography. Br. J. Sports Med. 1999, 33, 178-185. [CrossRef] [PubMed] 
55. Santalla, A.; Pérez, M.; Montilla, M.; Vicente, L.; Davison, R.; Earnest, C.; Lucía, A. Sodium bicarbonate ingestion does not alter the slow component of oxygen uptake kinetics in professional cyclists. J. Sports Sci. 2003, 1, 39-47. [CrossRef]

56. Jones, L.W.; Liang, Y.; Pituskin, E.N.; Battaglini, C.L.; Scott, J.M.; Hornsby, W.E.; Haykowsky, M. Effect of Exercise Training on Peak Oxygen Consumption in Patients with Cancer: A Meta-Analysis. Oncologist 2011, 16, 112-120. [CrossRef] [PubMed]

57. Clerc, P.; Rigoulet, M.; Leverve, X.; Fontaine, E. Nitric oxide increases oxidative phosphorylation efficiency. J. Bioenerg. Biomembr. 2007, 39, 158-166. [CrossRef] [PubMed]

58. Faude, O.; Kindermann, W.; Meyer, T. Lactate threshold concepts. Sports Med. 2009, 39, 469-490. [CrossRef] [PubMed]

59. Beaver, W.L.; Wasserman, K.; Whipp, B.J. A new method for detecting anaerobic threshold by gas exchange. J. Appl. Physiol. 1986, 60, 2020-2027. [PubMed]

60. Galler, S.; Hilber, K.; Gobesberger, A. Effects of nitric oxide on force-generating proteins of skeletal muscle. Pflug. Arch. 1997, 434, 242-245. [CrossRef] [PubMed]

61. Bescós, R.; Sureda, A.; Tur, J.A.; Pons, A. The effect of Nitric-Oxide-related supplements on human performance. Sports Med. 2012, 42, 99-117. [CrossRef] [PubMed]

62. Hernández, A.; Schiffer, T.A.; Ivarsson, N.; Cheng, A.J.; Bruton, J.D.; Lundberg, J.O.; Weitzberg, E.; Westerblad, H. Dietary nitrate increases tetanic $\left[\mathrm{Ca}^{2+}\right]_{i}$ and contractile force in mouse fast-twitch muscle. J. Physiol. 2012, 590, 3575-3583. [CrossRef] [PubMed]

63. Koehle, M.S.; Cheng, I.; Sporer, B. Canadian academy of sport and exercise medicine position statement: Athletes at high altitude. Clin. J. Sport Med. 2014, 24, 120-127. [CrossRef] [PubMed]

64. Droma, Y.; Hanaoka, M.; Ota, M.; Katsuyama, Y.; Koizumi, T.; Fujimoto, K.; Kobayashi, T.; Kubo, K. Positive association of the endothelial nitric oxide synthase gene polymorphisms with highaltitude pulmonary edema. Circulation 2002, 106, 826-830. [CrossRef] [PubMed]

65. Duplain, H.; Sartori, C.; Lepori, M.; Eqli, M.; Alemann, Y.; Nicod, P.; Scherrer, U. Exhaled nitric oxide in highaltitude pulmonary edema: Role in the regulation of pulmonary vascular tone and evidence for a role against inflammation. Am. J. Respir. Crit. Care Med. 2000, 162, 221-224. [CrossRef] [PubMed]

66. Casey, D.P.; Madery, B.D.; Curry, T.B.; Eisenach, J.H.; Wilkins, B.W.; Joyner, M.J. Nitric oxide contributes to the augmented vasodilation during hypoxic exercise. J. Physiol. 2010, 588, 373-385. [CrossRef] [PubMed]

67. Hoffman, J.R.; Kang, J.; Ratamess, N.A.; Jennings, P.F.; Mangine, G.T.; Faigenbaum, A.D. Effect of nutritionally enriched coffee consumption on aerobic and anaerobic exercise performance. J. Strength Cond. Res. 2007, 21, 456-459. [CrossRef] [PubMed]

68. Stear, S.J.; Castell, L.M.; Burke, L.M.; Spriet, L.L. BJSM reviews: A-Z of supplements: Dietary supplements, sports nutrition foods and ergogenic aids for health and performance-Part 6. Br. J. Sports Med. 2010, 44, 297-308. [CrossRef] [PubMed]

69. Williams, J.H. Caffeine, neuromuscular function and high-intensity exercise performance. J. Sports Med. Phys. 1991, 31, 481-489.

70. Magkos, F.; Kavouras, S.A. Caffeine use in sports, pharmacokinetics in man, and cellular mechanisms of action. Crit. Rev. Food Sci. Nutr. 2005, 45, 535-562. [CrossRef] [PubMed]

71. Goldstein, E.; Jacobs, P.L.; Whiterhurst, M.; Penhollow, T.; Antonio, J. Caffeine airnticele enhances upper body strength in resistance-trained women. J. Int. Soc. Sports Nutr. 2010, 14, 7-18.

72. Bell, D.G.; McLellan, T.M. Exercise endurance 1, 3, and $6 \mathrm{~h}$ after caffeine ingestion in caffeine users and nonusers. J. Appl. Physiol. 2002, 93, 1227. [CrossRef] [PubMed]

73. Schneiker, K.T.; Bishop, D.; Dawson, B.; Hackett, L.P. Effects of caffeine on prolonged intermittent-sprint ability in team-sport athletes. Med. Sci. Sports Exerc. 2006, 38, 578-585. [CrossRef] [PubMed]

74. Stuart, G.R.; Hopkins, W.G.; Cook, C.; Cairns, S.P. Multiple effects of caffeine on simulated high-intensity team-sport performance. Med. Sci. Sports Exerc. 2005, 37, 1998-2005. [CrossRef] [PubMed]

75. Desbrow, B.; Biddulph, C.; Devlin, B.; Grant, G.D.; Anoopkumar-Dukie, S.; Leveritt, M.D. The effects of different doses of caffeine on endurance cycling time trial performance. J. Sports Sci. 2012, 30, 115-120. [CrossRef] [PubMed]

76. Webb, A.J.; Patel, N.; Loukogeorgakis, S.; Okorie, M.; Aboud, Z.; Misra, S.; Rashid, R.; Miall, P.; Deanfield, J.; Benjamin, N. Acute blood pressure lowering, vasoprotective, and antiplatelet properties of dietary nitrate via bioconversion to nitrite. Hypertension 2008, 51, 784-790. [CrossRef] [PubMed] 
77. Govoni, M.; Jansson, E.A.; Weitzberg, E.; Lundberg, J.O. The increase in plasma nitrite after a dietary nitrate load is markedly attenuated by an antibacterial mouthwash. Nitric Oxide 2008, 19, 333-337. [CrossRef] [PubMed]

78. Jones, A.M. Dietary Nitrate Supplementation and Exercise Performance. Sports Med. 2014, 44, 35-45. [CrossRef] [PubMed]

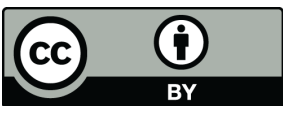

(C) 2017 by the authors; licensee MDPI, Basel, Switzerland. This article is an open access article distributed under the terms and conditions of the Creative Commons Attribution (CC-BY) license (http://creativecommons.org/licenses/by/4.0/). 